\title{
Quantum correlated heat engine with spin squeezing
}

\author{
Ferdi Altintas, ${ }^{1}$ Ali Ü. C. Hardal, ${ }^{2}$ and Özgür E. Müstecaplıog̃lu ${ }^{2, *}$ \\ ${ }^{1}$ Department of Physics, Abant Izzet Baysal University, Bolu 14280, Turkey \\ ${ }^{2}$ Department of Physics, Koç University, Ístanbul, Sartyer 34450, Turkey
}

(Received 20 March 2014; revised manuscript received 30 June 2014; published 2 September 2014)

\begin{abstract}
We propose a four-level quantum heat engine in an Otto cycle with a working substance of two spins subject to an external magnetic field and coupled to each other by a one-axis twisting spin squeezing nonlinear interaction. We calculate the positive work and the efficiency of the engine for different parameter regimes. In particular, we investigate the effects of quantum correlations at the end of the two isochoric processes of the Otto cycle, as measured by the entanglement of formation and quantum discord, on the work extraction and efficiency. The regimes where the quantum correlations could enhance the efficiency and work extraction are characterized.
\end{abstract}

DOI: 10.1103/PhysRevE.90.032102

PACS number(s): 05.30.-d, 05.70.-a, 03.65.Ud, 03.67.Mn

\section{INTRODUCTION}

Heat engines are crucial tools for our modern society, and their miniaturization is required for our further development beyond the industrial era. Recent progress in producing and controlling systems in micro-, nano-, and even in atomiclength scales inspired many proposals of heat engines which could operate in the quantum realm. Such engines are called quantum heat engines (QHEs), and despite their small length scales they promise surprisingly high efficiency [1-10]. Some intriguing QHEs are quantum information engines which could exploit quantum coherence as a resource to harvest useful work more efficiently than the Carnot limit, without violating the second law of thermodynamics $[2,9]$. In addition to the appealing practical value of implementing such miniature quantum machines, their fundamental studies could extend thermodynamics to the quantum regime and establish its place there from the perspective of quantum information theory.

We propose here a QHE with a working substance of a pair of spins subject to an external magnetic field and coupled to each other by a so called one-axis twisting nonlinear spin squeezing interaction [11-13]. The motivation behind considering this particular interaction is its capability to establish pairwise quantum correlations among the spins. The engine is assumed to operate in a quantum Otto cycle [7,8] that consists of two adiabatic and two isochoric stages. Our first objective is to examine the work extraction out of the engine and its efficiency by changing either the external field or spin-spin interaction strength in the adiabatic stages.

Our second objective is to investigate how the quantum correlations play a role in the performance of the engine. The quantum state of the working medium at the end of the two isochoric processes can be represented by a canonical ensemble. Since the temperature of the heat baths as well as the system parameters can be externally controlled [14,15], it would also be possible to control the amount of quantum correlations of the working substance in the two heat baths. We discuss the quantum correlations in our system by analyzing the behavior of the entanglement of formation (EoF) [16] and quantum discord (QD) [17,18]. QD can measure quantum

*omustecap@ku.edu.tr correlations beyond entanglement (or "quantumness") and more robust than entanglement in the presence of thermal noise [19]. We would like to explore if quantum correlations and entanglement are mere by-products of the QHE or if they can enhance its performance. The general answer to this question remained to be elusive so far and model-dependent effects are reported, as we shall summarize below. Our choice of spin squeezing nonlinear interaction model is on purpose to establish strong bipartite quantum correlations and entanglement in the QHE.

Along similar lines to ours, quantum entanglement was studied as a quantum resource in QHEs [9,10,20-24], and its positive or negative effects on the engine performance is found to be model dependent. In particular, work cannot be extracted in some coupled spin models, if the entanglement of spins in the hot bath exceeds the one in the cold bath $[9,10,20,21]$. Conversely, entanglement could enhance the performance of some quantum machines [23-26]. QD was investigated in the context of quantum absorption chillers for a particular model of interacting three qubits (two-level systems), albeit its role on the performance of the quantum refrigerator remained elusive and unclear [27].

Our analysis revealed the parameter regimes in our model for which QD or entanglement can be interpreted as a resource. We find that entanglement of the working substance at the end of hot bath stage of the Otto cycle forbids the work extraction, if the external field or the interactions are stronger in hot bath stage relative to cold bath stage. In this case, QD at the end of cold bath stage is constructive for positive work and efficiency over a wide range of interaction strengths, while QD at the hot bath stage can be either constructive or destructive depending on the system parameters. Their difference is constructive provided that QD is greater at the cold bath stage. When the interaction is weaker in the hot bath stage, both the entanglement and QD lead to enhanced work and efficiency.

This article is organized as follows. In Sec. II we describe our two-spin QHE by describing the working substance, its thermalization, and the Otto cycle. In Sec. III we summarize the quantum work, efficiency, and quantum correlation measures which we calculate. The results are reported and discussed in Sec. IV. A comparison of our results with relevant previous studies is given in Sec. V. We present our conclusions in Sec. VI. 


\section{MODEL SYSTEM: THE QUANTUM OTTO ENGINE}

\section{A. Working substance}

We consider a four-level quantum Otto engine with a working medium of two spins under an external magnetic field and coupled to each other by a one-axis twisting spin squeezing interaction. The Hamiltonian of the working substance can be written as [11,12]

$$
H=\mu S_{x}^{2}+\Omega S_{z},
$$

where $\Omega(\geqslant 0)$ is the strength of the external magnetic field in the $z$ direction, $\mu(\geqslant 0)$ describes the strength of the spin squeezing interaction in the $x$ direction, $S_{\alpha}=\frac{1}{2} \sum_{i=1}^{2} \sigma_{\alpha}^{(i)}$, $(\alpha \in\{x, y, z\})$ are the collective spin operators, and $\sigma_{\alpha}^{(i)}$ are the Pauli matrices for the $i$ th spin.

Quadratic nonlinearity is the only polynomial nonlinearity our system can have as $\sigma_{\alpha}^{(i) 2}=\mathbb{1}_{i}$. Even powers of the collective spin $S_{x}^{n}$ with $n=4,6,8, \ldots$ are the same with $S_{x}^{2}$ while the odd powers $S_{x}^{m}$ with $m=3,5,7, \ldots$ are the same with $S_{x}$. A term of the form $\mu S_{x}$ in the Hamiltonian is just a rotation operation and cannot induce quantum correlations between the spins. The spin squeezing interaction $\mu S_{x}^{2}$ on the other hand establishes pairwise correlations between the individual spins in a collective spin system; in our case bipartite correlations are formed between the two spins.

For a system of two spins, the eigenvalues $E_{n}$ and the corresponding eigenvectors $\left|\Psi_{n}\right\rangle$ of the Hamiltonian (1) can be calculated as

$$
\begin{aligned}
E_{1,4} & =\frac{\mu \mp \kappa}{2}, \quad\left|\Psi_{1}\right\rangle=\frac{1}{A_{\mp}}(\mu|00\rangle+(2 \Omega \mp \kappa)|11\rangle), \\
E_{2} & =0, \quad\left|\Psi_{2}\right\rangle=\frac{1}{\sqrt{2}}(|10\rangle-|01\rangle), \\
E_{3} & =\mu, \quad\left|\Psi_{3}\right\rangle=\frac{1}{\sqrt{2}}(|10\rangle+|01\rangle),
\end{aligned}
$$

where $\kappa=\sqrt{\mu^{2}+4 \Omega^{2}}$ and $A_{ \pm}=\sqrt{\mu^{2}+(2 \Omega \pm \kappa)^{2}}$. The eigenvalues are plotted in Fig. 1.

\section{B. Thermalization}

When thermal fluctuations are introduced into the system, the reduced density matrix of the working substance at thermal equilibrium with the heat bath can be written as

$$
\rho=\frac{1}{Z} e^{-\beta H}=\sum_{n=1}^{4} P_{n}(T)\left|\Psi_{n}\right\rangle\left\langle\Psi_{n}\right|,
$$

where $P_{n}(T)=e^{-\beta E_{n}} / Z$ are the occupation probabilities of the eigenstates, $\beta=1 / k_{B} T\left(k_{B}=1\right), T$ is the temperature, and $Z=\sum_{n} e^{-\beta E_{n}}$ is the partition function.

Thermalization is not a trivial assumption. In order to verify it we examine the time evolution of our system when it is in contact with a heat reservoir. There could be different thermalization strategies depending on the actual physical system implementation. We consider here a typical scenario where two spins are coupled to two separate heat reservoirs of quantum oscillators. We assume both reservoirs lead to same decoherence rates $\gamma$, and they are at the same temperature $T$. We assume energy parameters such as $\Omega, \mu$ are dimensionless
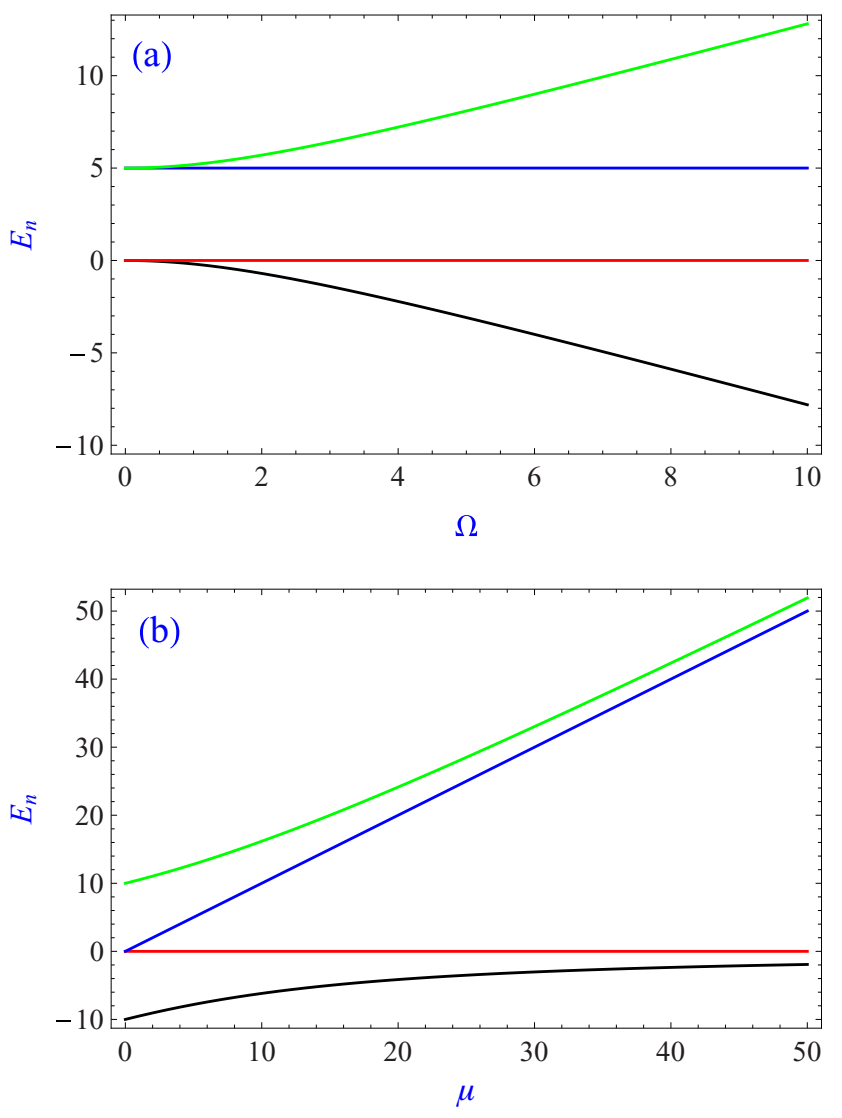

FIG. 1. (Color online) The energy spectrum $E_{n}(n=1,2,3,4$ from bottom to top, respectively) versus $\Omega$ for $\mu=5$ (a), and versus $\mu$ for $\Omega=10$ (b)

and scaled by $\Omega$. Temperature is also dimensionless and scaled by $\Omega / k_{B}$. The average number of oscillator quanta in either reservoirs is given by $\bar{n}=1 /[\exp (\Omega / T)-1]$. Dynamics of the system can be determined by a master equation under Born and Markov approximations [28],

$$
\begin{aligned}
\frac{d \rho}{d t}= & -\mathrm{i}[H, \rho]-\frac{\gamma}{2}\left[(\bar{n}+1) \sum_{i=1}^{2}\left(\sigma_{+}^{(i)} \sigma_{-}^{(i)} \rho-\sigma_{-}^{(i)} \rho \sigma_{+}^{(i)}\right)\right. \\
& \left.+\bar{n} \sum_{i=1}^{2}\left(\sigma_{-}^{(i)} \sigma_{+}^{(i)} \rho-\sigma_{+}^{(i)} \rho \sigma_{-}^{(i)}\right)+\text { H.c. }\right],
\end{aligned}
$$

where time $t$ is dimensionless and scaled by $1 / \Omega$. The time-dependent density matrix $\rho$ is determined for an initial state $|10\rangle$ using the master equation for various $\gamma$; then it is compared with the thermal density matrix $\rho_{\text {th }}$ in Eq. (3) by evaluating the mixed state fidelity $F\left(\rho_{\mathrm{th}}, \rho\right)=\left[\operatorname{Tr}\left(\sqrt{\sqrt{\rho_{\mathrm{th}}} \rho \sqrt{\rho_{\mathrm{th}}}}\right)\right]^{2}$ [29]. Our results are shown in Fig. 2 where we take $\Omega=$ $4, \mu=2.5, T=4$, and $\gamma=0.001,0.005,0.001,0.05$. At the temperature $T=4$, the average quanta of oscillators in the heat reservoirs is $\bar{n} \sim 0.6$. We see that thermalization can be achieved at shorter times with increasing decoherence rate $\gamma$ with high fidelity close to 1 which verifies the thermalization assumption for our system. 


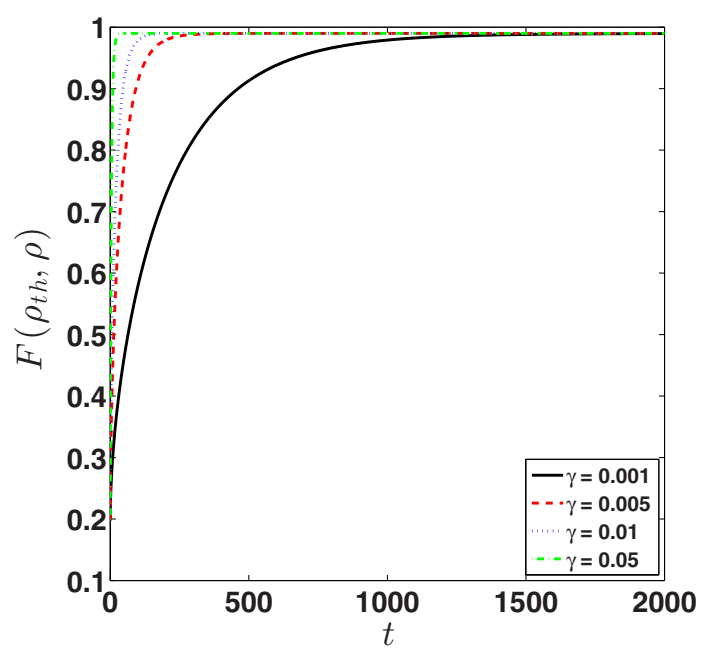

FIG. 2. (Color online) Fidelity $F\left(\rho_{\text {th }}, \rho\right)$ of the time-dependent density matrix $\rho$ to the thermal density matrix $\rho_{\text {th }} . \rho$ is determined by the master equation evolution of an initial state $|10\rangle$ for $\Omega=4, \mu=$ $2.5, T=4$, and $\gamma=0.001,0.005,0.001,0.05$. Time $t$ and fidelity $F\left(\rho_{\text {th }}, \rho\right)$ are dimensionless. Time and energy are scaled by $1 / \Omega$ and $\Omega$, respectively, as explained in the text. Thermalization is achieved at shorter time with increasing decoherence rate $\gamma$ with high fidelity.

\section{Quantum Otto cycle}

The stages of the proposed quantum Otto engine can be described as follows:

Step 1: The working substance, having energy levels $E_{n}^{H}$ and initial probabilities in each eigenstates, is brought into contact with a hot heat bath at $T=T_{H}$. Upon attaining thermal equilibrium with the heat bath, the occupation probabilities change to $P_{n}\left(T_{H}\right)$, while the energy levels remain the same. An amount of heat is absorbed, but no work is done during this quantum isochoric process.

Step 2: The working medium is isolated from the heat bath and exposed to the quantum adiabatic expansion process, in which the energy structure is changed from $E_{n}^{H}$ to $E_{n}^{L}$. Provided the change of the energies is slow enough, the occupation probabilities are maintained according to the quantum adiabatic theorem. An amount of work is done, but no heat is exchanged.

Step 3: The working substance goes through another quantum isochoric process where it is brought into contact with a cold heat bath at $T=T_{L}\left(T_{H}>T_{L}\right)$. After the thermalization process, an amount of heat is released but no work is done. At the end of the stage, the occupation probabilities become $P_{n}\left(T_{L}\right)$, while the energy levels, $E_{n}^{L}$, remain unchanged.

Step 4: The system is removed from the cold heat bath and undergoes quantum adiabatic contraction process, which changes the energy levels from $E_{n}^{L}$ to $E_{n}^{H}$, but keeps the probabilities the same. An amount of work is done during the stage, but no heat is exchanged. At the end of this stage, the working medium returns to its initial condition and is ready to cycle again.

We will consider two different schemes for the the adiabatic steps: (a) the magnetic field is altered between two values $\left(\Omega_{H} \rightarrow \Omega_{L} \rightarrow \Omega_{H}\right)$ at a fixed interaction coefficient $\mu$, and (b) the squeezing coefficient is changed between two values $\left(\mu_{H} \rightarrow \mu_{L} \rightarrow \mu_{H}\right)$ at a fixed magnetic field $\Omega$.

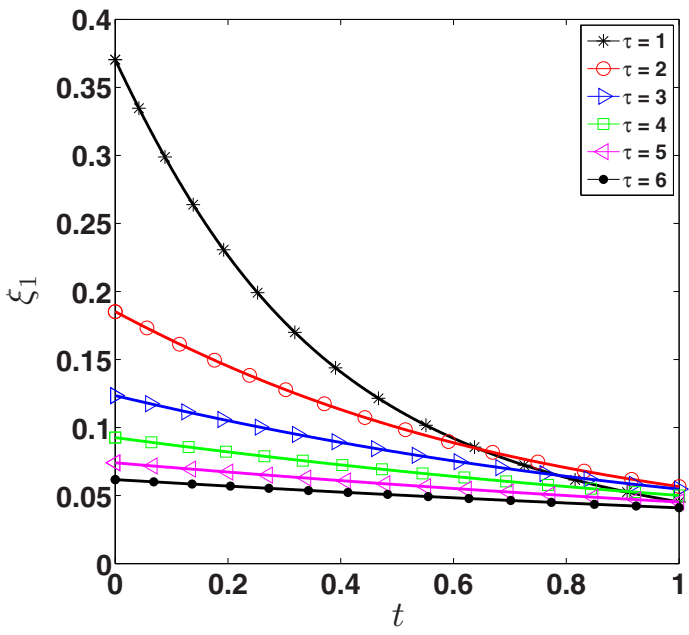

FIG. 3. (Color online) Time dependence of the adiabaticity parameter $\xi_{1}$. Adiabatic evolution requires $\xi_{1} \ll 1$. We take $\Omega_{H} / \Omega_{L}=4$, $\mu=2.7$, and $\tau=0.1,0.2, \ldots, 0.6$. Time $t$ and $\xi_{1}$ are dimensionless. Time and energy are scaled by $1 / \Omega_{L}$ and $\Omega_{L}$, respectively, as explained in the text. Adiabatic condition can be satisfied more strongly at higher $\tau$ due to slower change of $\Omega_{L}$ to $\Omega_{H}$.

\section{Quantum adiabaticity}

Our Otto cycle relies on validity of thermalization and quantum adiabatic evolution assumptions. We already discussed the thermalization in our system, and now let us verify the adiabaticity condition given by [30]

$$
\xi_{n}=\sum_{m \neq n}\left|\frac{\left\langle\psi_{m}(t) \mid \dot{\psi}_{n}(t)\right\rangle}{E_{m}(t)-E_{n}(t)}\right|,
$$

where $n, m=1,2,3,4$. Quantum adiabatic theorem requires $\xi_{n} \ll 1$. The instantaneous eigenvectors $\left|\psi_{n}(t)\right\rangle$ and eigenvalues $E_{n}(t)$ are determined by time-dependent Hamiltonian $H(t)=\Omega(t) S_{z}+\mu S_{x}^{2}$ with

$$
\Omega(t)=\Omega_{L}+\frac{\Omega_{H}-\Omega_{L}}{\tau} t,
$$

where $\tau$ is the duration of the adiabatic stage where $\Omega_{L}$ is changed to $\Omega_{H}$.

There could be other schemes to change $\Omega_{L}$ to $\Omega_{H}$; we consider a simple form to verify the possibility of adiabatic evolution. We illustrate the validity of adiabaticity for the case of altering $\Omega$ during the adiabatic stages. A similar method can be used to verify the case of changing $\mu$. The eigensystem of our model yields $\xi_{2}=\xi_{3}=0$ and $\xi_{1}=\xi_{4}$. We assume the energy $\Omega, \mu$ and time $t$ parameters are dimesionless and scaled by $\Omega_{L}$ and $1 / \Omega_{L}$, respectively. The time dependence of $\xi_{1}$ is shown in Fig. 3 where we take $\Omega_{H} / \Omega_{L}=4, \mu=2.7$, and $\tau=$ $0.1,0.2, \ldots, 0.6$. We see that adiabaticity condition is satisfied at all times for these cases. The condition is better satisfied for longer processes with higher $\tau$ as the change from $\Omega_{L}$ to $\Omega_{H}$ happens more slowly with smaller slope in Eq. (6). We numerically found that rapid change of $\Omega$ with $\tau<0.5$ yields $\xi_{1}>1$ during early stages of evolution and the adiabaticity condition fails. 


\section{THEORY}

\section{A. Work and efficiency}

The heat transfer and the work performed at the quantum level can be calculated through the interpretation of the quantum first law of thermodynamics [7,8]: $d U=d Q+d W=$ $\sum_{n}\left\{E_{n} d P_{n}+P_{n} d E_{n}\right\}$. In this interpretation, the infinitesimal heat transfer, $d Q=\sum_{n} E_{n} d P_{n}$, is associated with the change of occupation probabilities, and the infinitesimal work done, $d W=\sum_{n} P_{n} d E_{n}$, is with the change in energy levels. The heat absorbed, $Q_{\text {in }}$, during stage 1 , the heat released, $Q_{\text {out }}$, during stage 3 , the net work done, $W$, and the operational efficiency, $\eta$, of the engine can be obtained easily through this interpretation $[7,8]$ :

$$
\begin{aligned}
Q_{\text {in }} & =\sum_{n} E_{n}^{H}\left[P_{n}\left(T_{H}\right)-P_{n}\left(T_{L}\right)\right], \\
Q_{\text {out }} & =\sum_{n} E_{n}^{L}\left[P_{n}\left(T_{L}\right)-P_{n}\left(T_{H}\right)\right], \\
W & =Q_{\text {in }}+Q_{\text {out }} \\
& =\sum_{n}\left[E_{n}^{H}-E_{n}^{L}\right]\left[P_{n}\left(T_{H}\right)-P_{n}\left(T_{L}\right)\right], \\
\eta & =\frac{W}{Q_{\text {in }}},
\end{aligned}
$$

where $E_{n}^{H}\left(E_{n}^{L}\right)$ are the energy levels during the stage 1 (3), which can be obtained by replacing $\mu$ and $\Omega$ by $\mu_{H}\left(\mu_{L}\right)$ and $\Omega_{H}\left(\Omega_{L}\right)$, respectively. For the positive work extraction $(W>0)$, the physically acceptable situation is considered, i.e., $Q_{\text {in }}>-Q_{\text {out }}>0$. The possible cases, $Q_{\text {in }}>Q_{\text {out }}>0$ and $Q_{\text {out }}>-Q_{\text {in }}>0$, which violate the second law of thermodynamics are excluded in the present study. Moreover, we have considered the efficiency of the engine when $W>0$.

We will discuss the work extraction and efficiency by considering two different schemes for the the adiabatic steps: (a) the magnetic field is altered between two values $\left(\Omega_{H} \rightarrow \Omega_{L} \rightarrow \Omega_{H}\right)$ at a fixed interaction coefficient $\mu$, and (b) the squeezing coefficient is changed between two values $\left(\mu_{H} \rightarrow \mu_{L} \rightarrow \mu_{H}\right)$ at a fixed magnetic field $\Omega$. In both cases, we will also discuss the possibility of work extraction in two parameter regions: (i) $\Omega_{H}>\Omega_{L}$ and (ii) $\Omega_{H}<\Omega_{L}$ for the case (a), and (i) $\mu_{H}>\mu_{L}$ and (ii) $\mu_{H}<\mu_{L}$ for the case (b).

\section{B. Quantum discord and entanglement}

We will also study the role of quantum correlations as measured by quantum discord and entanglement on the thermodynamic quantities for these cases. First, we note that the thermal density matrix in the standard basis $\{|1\rangle \equiv$ $|11\rangle,|2\rangle \equiv|10\rangle,|3\rangle \equiv|01\rangle,|4\rangle \equiv|00\rangle\}$ given in Eq. (3) has matrix elements in the following form:

$$
\rho=\left(\begin{array}{cccc}
a & 0 & 0 & w \\
0 & b & z & 0 \\
0 & z & b & 0 \\
w & 0 & 0 & d
\end{array}\right)
$$

The matrix elements can be easily determined by using Eqs. (2) and (3). The calculation of QD requires a maximization procedure over projective measurements performed locally on one system, which cannot be done analytically in general and it is computationally inefficient [31]. However, for the above density matrix, the extremization in the definition of quantum discord can be done analytically [32] up to a small worst-case absolute error [33]. The analytic form of the QD can be written as [32]

$$
D=\min \left\{D_{1}, D_{2}\right\}
$$

where

$$
\begin{aligned}
D_{1}= & S\left(\rho_{A}\right)-S(\rho)-a \log _{2}\left(\frac{a}{a+b}\right)-b \log _{2}\left(\frac{b}{a+b}\right) \\
& -d \log _{2}\left(\frac{d}{b+d}\right)-b \log _{2}\left(\frac{b}{d+b}\right) \\
D_{2}= & S\left(\rho_{A}\right)-S(\rho)-\Delta_{+} \log _{2} \Delta_{+}-\Delta_{-} \log _{2} \Delta_{-}
\end{aligned}
$$

where $\Delta_{ \pm}=\frac{1}{2}(1 \pm \Gamma), \Gamma^{2}=(a-d)^{2}+4(|z|+|w|)^{2}, \rho_{A}=$ $\operatorname{Tr}_{B} \rho$ is the density matrix of subsystem $A$, and $S(\rho)=$ $-\operatorname{Tr}\left(\rho \log _{2} \rho\right)$ is the von Neumann entropy. Since $S\left(\rho_{A}\right)=$ $S\left(\rho_{B}\right)$ for the density matrix (8), the projective measurement performed on the subsystem $\mathrm{A}$ or $\mathrm{B}$ assumes equal values. The definition of QD emerges from the mismatch of two expressions of mutual information extended from classical to quantum systems and is basically defined as the difference between total correlations measured by mutual information and the classical correlations determined by measurement-based conditional entropy. Nonzero QD indicates the impossibility of accessing all information about one subsystem by performing a set of measurements on the other subsystem.

Similarly, as an entanglement measure, EoF for the density matrix can be written as [16]

$$
E=h\left[\frac{1}{2}\left(1+\sqrt{1-C^{2}}\right)\right],
$$

where $h[x]=-x \log _{2} x-(1-x) \log _{2}(1-x)$ and $C=$ $2 \max \{0,|z|-\sqrt{a d},|w|-b\}$ is the concurrence for the density matrix (8). EoF is one of the well-motivated measures of the degree of entanglement for bipartite states. EoF and QD have the same entropic interpretation in their definitions, being equal for pure states and having a strict connection by monogamic relations for mixed states. So the two figures of merit are the natural choices for a quantitative comparison of quantum correlations. However, we should stress here that they quantify a different part of quantum correlations for mixed states; there are separable mixed states with nonnull QD. In fact, QD is nonzero for almost all mixed quantum states [34].

\section{RESULTS}

In the following we will denote QD and EoF as $D_{H}\left(D_{L}\right)$ and $E_{H}\left(E_{L}\right)$ for the hot (cold) heat bath cases, respectively. We shall calculate the thermodynamical work and operational efficiency and briefly discuss the temperature effects on them as well as on quantum correlations.

If we change the applied magnetic field $(\Omega)$ in the adiabatic stages, we get different work and efficiency out of the engine depending on the different amount of interaction $(\mu)$ between the spins. We would like to see how quantum correlations build up depending on the interaction in comparison to the work output and efficiency. In addition we ask the same question if 

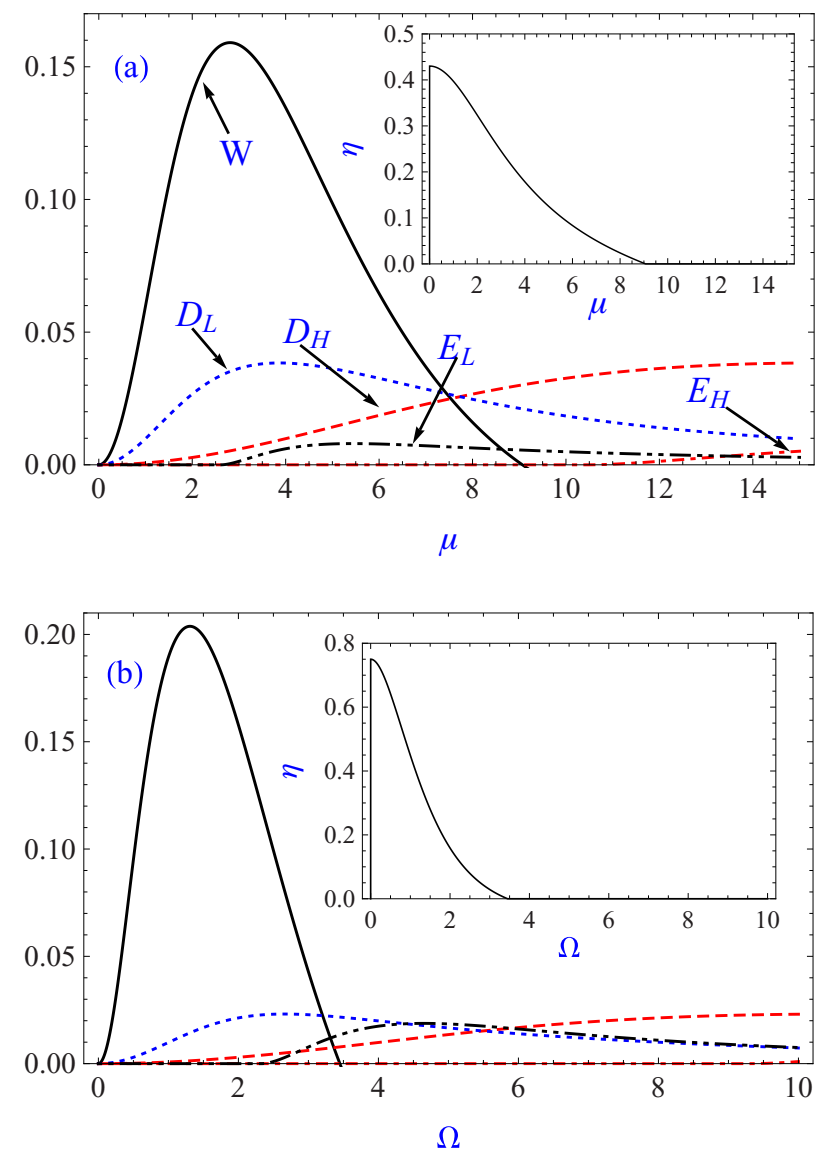

FIG. 4. (Color online) Work (solid, line), efficiency (insets), $D_{H}$ (red, dashed line), $D_{L}$ (blue, dotted line), $E_{H}$ (red, dot-dashed line), and $E_{L}$ (black, dot-dot-dashed line) versus $\mu$ (a) with $\mu_{H}=\mu_{L}=\mu$, $\Omega_{H}=4, \Omega_{L}=1, k_{B} T_{H}=4$, and $k_{B} T_{L}=1$, and versus $\Omega$ (b) with $\Omega_{H}=\Omega_{L}=\Omega, \mu_{H}=4, \mu_{L}=1, k_{B} T_{H}=4$, and $k_{B} T_{L}=1$.

we vary the interaction in the adiabatic stages while keeping the $\Omega$ constant. For this purpose we plot the work, operational efficiency, and quantum correlation measures for these two cases in Fig. 4. In Fig. 4(a) we take $\mu_{H}=\mu_{L}=\mu, T_{H} / T_{L}=$ 4, and $\Omega_{H} / \Omega_{L}=4$, and in Fig. 4(b) we take $\Omega_{H}=\Omega_{L}=\Omega$ with $T_{H} / T_{L}=4$ and $\mu_{H} / \mu_{L}=4$. We obtain from Eqs. (2) and (7) that the efficiency and the positive work condition (PWC) for the case (a) with $\mu=0$ and for the case (b) with $\Omega=0$ have the usual formulas: (a) $\eta=1-\Omega_{L} / \Omega_{H}$ and $T_{H}>\left(\Omega_{H} / \Omega_{L}\right) T_{L}$ and (b) $\eta=1-\mu_{L} / \mu_{H}$ and $T_{H}>$ $\left(\mu_{H} / \mu_{L}\right) T_{L}$. Therefore, the engine does not produce work for the considered parameters in Fig. 4 with zero squeezing (a) and magnetic field (b), since the PWC is violated.

We see in Fig. 4(a) that the nonzero $\mu$ induces positive work. Work output first increases with $\mu$ until a critical value then drops to zero. Similarly, the nonzero $\Omega$ leads to positive work as in Fig. 4(b). Changing the magnetic field yields larger work and efficiency relative to changing interaction strength. The engine can operate close to the Carnot efficiency, $\eta_{C}=1-T_{L} / T_{H} \approx$ 0.75 as shown in the inset of Fig. 4(b). Efficiency decreases with $\mu$ and $\Omega$. There is optimal efficiency $\eta_{o}$ corresponding to maximum work, which is $\eta_{o} \approx 0.27$ at $\mu \approx 2.7$ for case (a) and $\eta_{0} \approx 0.34$ at $\Omega \approx 1.25$ for case (b). The work output increases with increasing efficiency for $\eta<\eta_{o}$.
At first glance, the quantum correlations, work, and efficiency relations in Fig. 4 have a complicated structure. On the other hand we can withdraw some general conclusions. In different models of spin systems it is reported that entanglement in the hot bath stage is detrimental for the work, and one cannot get work if entanglement is higher in the hot bath stage than the one in the cold bath stage [9,10,20,21]. Our engine indeed cannot produce work if it becomes entangled at the end of its contact with the hot bath; we find that $W=0$ when $E_{H}>0$ in Fig. 4. In addition it cannot operate with high efficiency, especially close to Carnot efficiency, if it is entangled in the cold bath stage, which is consistent with the results in Ref. [23]. $E_{L}$ and $E_{H}$ emerge just after the critical values of $\mu$ and $\Omega$.

On the other hand, quantum discord always exists, and our engine can actually work with high efficiency, even close to Carnot efficiency, with quantum correlations beyond entanglement. Figure 4 shows that work can be extracted when $D_{H}>0$ for both cases and even when $D_{H}>D_{L}$ in case (a). $W$ and $D_{L}$ have a nearly monotonic relation for the case (a). Entanglement in the cold bath stage is not detrimental for the operation of the engine, though it is not strong as much as quantum discord. We can conclude that the quantum correlations beyond entanglement in both cold and heat bath cases can play a constructive role on the performance of the heat engine. The following analysis will explore this conclusion further, and at the end we will focus more on the positive effects of quantum discord in the cold bath stage on the efficiency and work extraction.

We analyze the effect of the hot bath temperature and external field on the positive work, efficiency, and quantum correlations in the case of changing interaction strength during the adiabatic stages. We have also performed a similar analysis for the case where the external field is changed at the adiabatic stages and found similar qualitative results. Therefore, those results are not displayed here. Figure 5 shows that both work and efficiency increase with $T_{H}$. We see from Fig. 5(a) that the monotonic increase in work with $T_{H}$ is in an expected form. Higher $T_{H}$ for a given $T_{L}$ leads to more heat absorption, and more work is produced. External field increases the work output relative to $\Omega=0$ case as in Fig. 5(a), while it lowers the efficiency and makes it slightly temperature dependent as in Fig. 5(b). $W$ increases up to a critical point then decreases with $\Omega$. Figure 4 is a special case of this general behavior observed in Fig. 5(a) where there is a critical $\Omega$ for which $W$ is maximum for given $T_{H}$ and $T_{L}$. The engine operates with a fixed $\eta=1-\left(\mu_{L} / \mu_{H}\right)$ at $\Omega=0$ and produces work just after the PWC, $T_{H}>\left(\mu_{H} / \mu_{L}\right) T_{L}$. The external field modifies the PWC and makes the engine operate at a lower $T_{H}$ compared to the $\Omega=0$ case.

The quantumness is expected to degrade with the temperature. Figure 5(a) confirms this expectation. $E_{H}$ decreases with $T_{H}$ and disappears at $T_{H} \approx 2 . E_{L}$ is always zero in $0 \leqslant \Omega \leqslant 2$. We conclude that the working substance is fully separable at the end of two heat bath stages. On the other hand, quantum correlations beyond entanglement can survive at higher temperatures. We calculated $D_{H}$ and $D_{L}$ and find that they can coexist with the positive work. In the parameter regime of Fig. 5(a), $D_{H}$ behaves qualitatively the same as $E_{H}$ while $D_{L}$ is constant. Both are small $D_{H}<0.01, D_{L}<0.02$ 

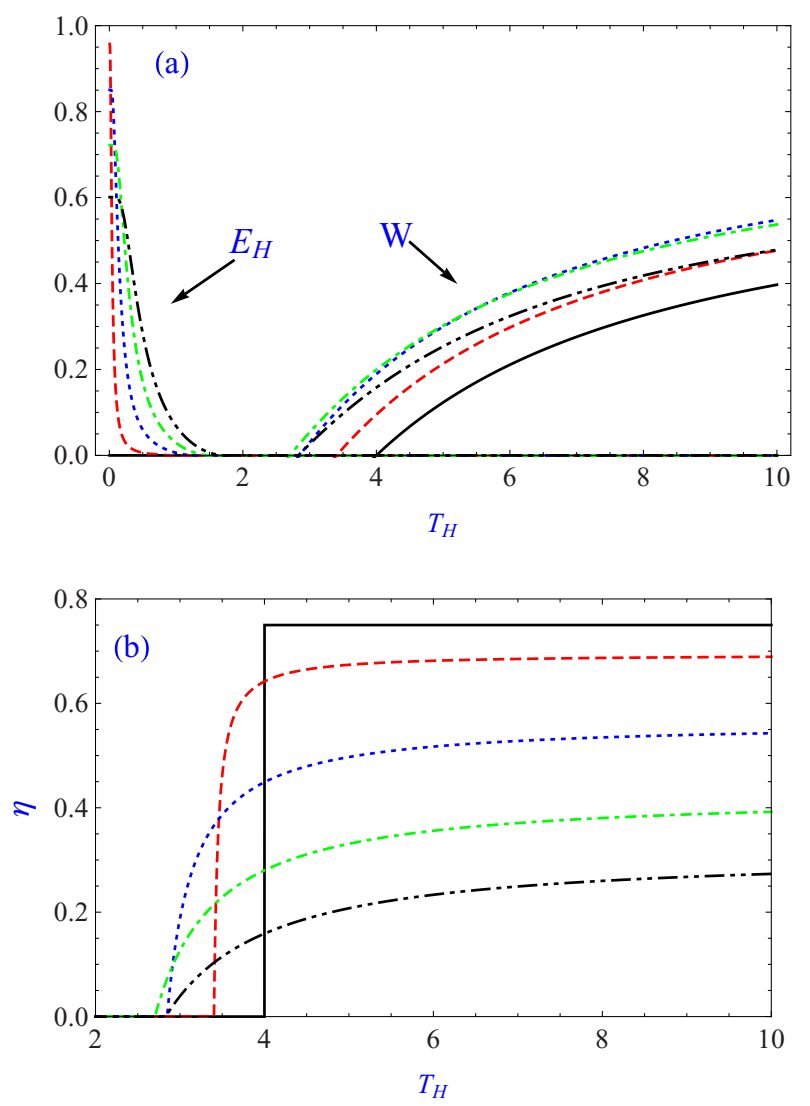

FIG. 5. (Color online) Dependence of entanglement of formation (EoF) $E_{H}$ [(a), right bunch], positive work $W$ [(a), left bunch], and efficiency $\eta$ (b) on the temperature of the hot reservoir $T_{H}$ (in units of $k_{B}$ ) for $k_{B} T_{L}=1, \mu_{H}=4, \mu_{L}=1, \Omega=0$ (black, solid line), $\Omega=0.5$ (red, dashed line), $\Omega=1$ (blue, dotted line), $\Omega=1.5$ (green, dot-dashed line), and $\Omega=2$ (black, dot-dot-dashed line). Note that for $\Omega=0$, EoF and quantum discord are both zero in the hot and cold bath stages.

but nonzero in the positive work regime. Figure 4 is an example of this situation at a particular set of $T_{H}$ and $T_{L}$.

Next, we analyze the effect of $\mu_{H}$ and $\Omega$ in the work extraction and the efficiency of the engine depicted in Fig. 6(a) and Fig. 6(b), respectively. If there is no external field, the strict conditions, $\mu_{H}>\mu_{L}$ and $T_{H}>\left(\mu_{H} / \mu_{L}\right) T_{L}$, determine the operation regime of the engine as $\mu_{L}<\mu_{H}<4 \mu_{L}$ in Fig. 6(a). The interval gets larger with $\Omega$. The efficiency increases until the Carnot point with $\mu_{H}$. In the region, $1<\mu_{H}<2.5$, the work output increases with the increasing efficiency. The nonzero $\Omega$ can lead to an increase of the work output at the cost of reduced efficiency.

For the temperature ranges in Fig. 6, the working medium has no entanglement $\left(E_{H}=E_{L}=0\right)$ at the end of the stages 1 and 3 of the engine cycle, while the quantum correlations beyond entanglement exist. $D_{H}$ increase with the interactions as shown in the inset of Fig. 6(a) while $D_{L}$ is independent of $\mu_{H}$ and hence is a nonzero constant depending on $\Omega$. There is a critical amount of quantum correlations indicated by $D_{H}$ at the maximum $W$ and $\eta$. Work output is larger but at a reduced efficiency relative to uncorrelated engine (QD and EoF are both zero) with $\Omega=0$. The quantum correlated Otto engine,
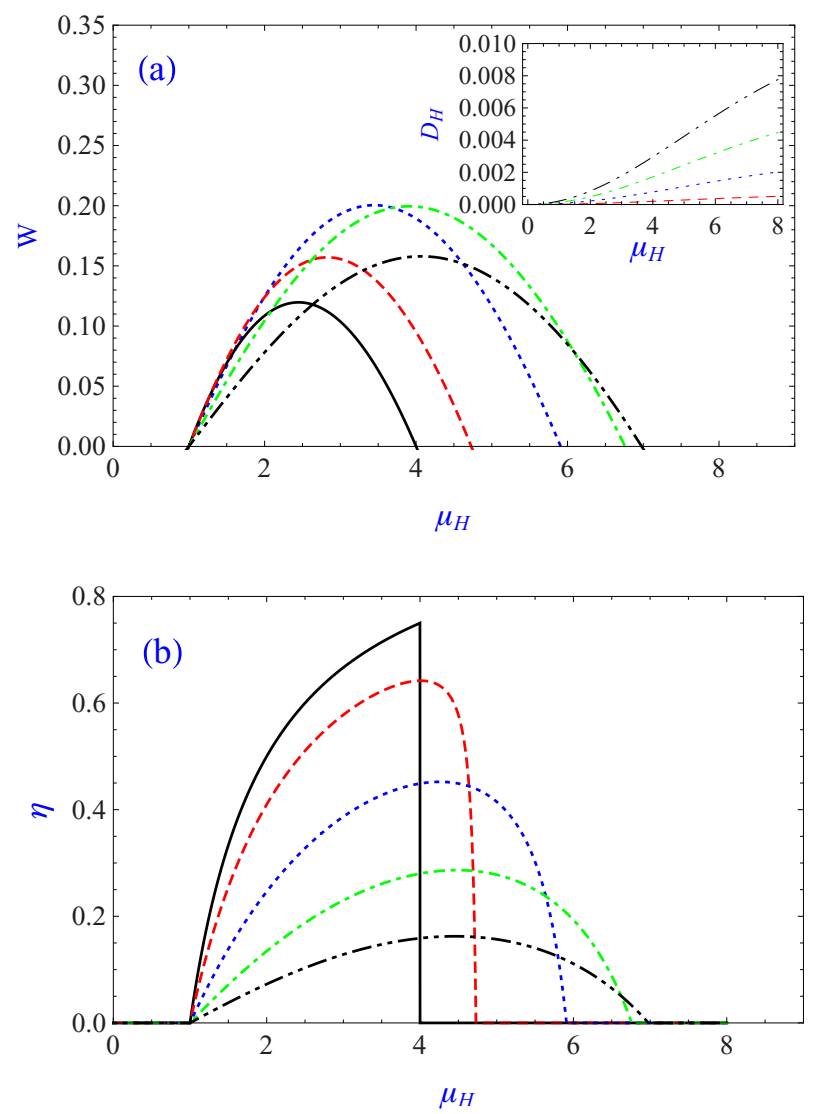

FIG. 6. (Color online) Dependence of positive work $W$ (a) and efficiency $\eta$ (b) on $\mu_{H}$ for $k_{B} T_{H}=4, k_{B} T_{L}=1, \mu_{L}=1, \Omega=0$ (black, solid line), $\Omega=0.5$ (red, dashed line), $\Omega=1$ (blue, dotted line), $\Omega=1.5$ (green, dot-dashed line), and $\Omega=2$ (black, dot-dotdashed line). The inset in (a) shows $D_{H}$ versus $\mu_{H}$ for the same parameters. Note that for $\Omega=0$, entanglement of formation and quantum discord are zero for both hot and cold bath stages.

with dominant correlations measured by QD, produces work in the parameter regimes $\left(\mu_{H}>4\right)$ where the uncorrelated engine $(\Omega=0)$ cannot. A similar analysis for the case where $\Omega$ is changed at the adiabatic stages produces similar results, and hence we have not presented them.

The energy gaps of the working substance play a subtle role in the performances of the quantum heat engines and determine the conditions in which work can be extracted efficiently $[8,35]$. For instance, if the system is noninteracting $\mu=0$, we have $E_{1,4}=\mp \Omega$ while $E_{2}=E_{3}=0$. The energy gaps get larger if $\Omega$ is increased from $\Omega_{H}$ to $\Omega_{L}$ in the second adiabatic stage of the Otto cycle. This would correspond to adiabatic contraction process, instead of expansion, and hence the engine could not produce work. This complies with the requirement of $\Omega_{L}<\Omega_{H}$ by the efficiency expression $\eta=1-\left(\Omega_{L} / \Omega_{H}\right)$. Similarly, if there is no external field $\Omega=$ 0 , then the level structure becomes $E_{1,3,4}=\mu$ and $E_{2}=0$. Increasing the interaction strength from $\mu_{H}$ to $\mu_{L}$ in the second stage again makes the adiabatic process a contraction rather than an expansion. The efficiency $\eta=1-\left(\mu_{L} / \mu_{H}\right)$ becomes negative, and the engine cannot produce work. The presence of an external field or interactions can change this picture during the variation of the control parameters in the adiabatic stages. 

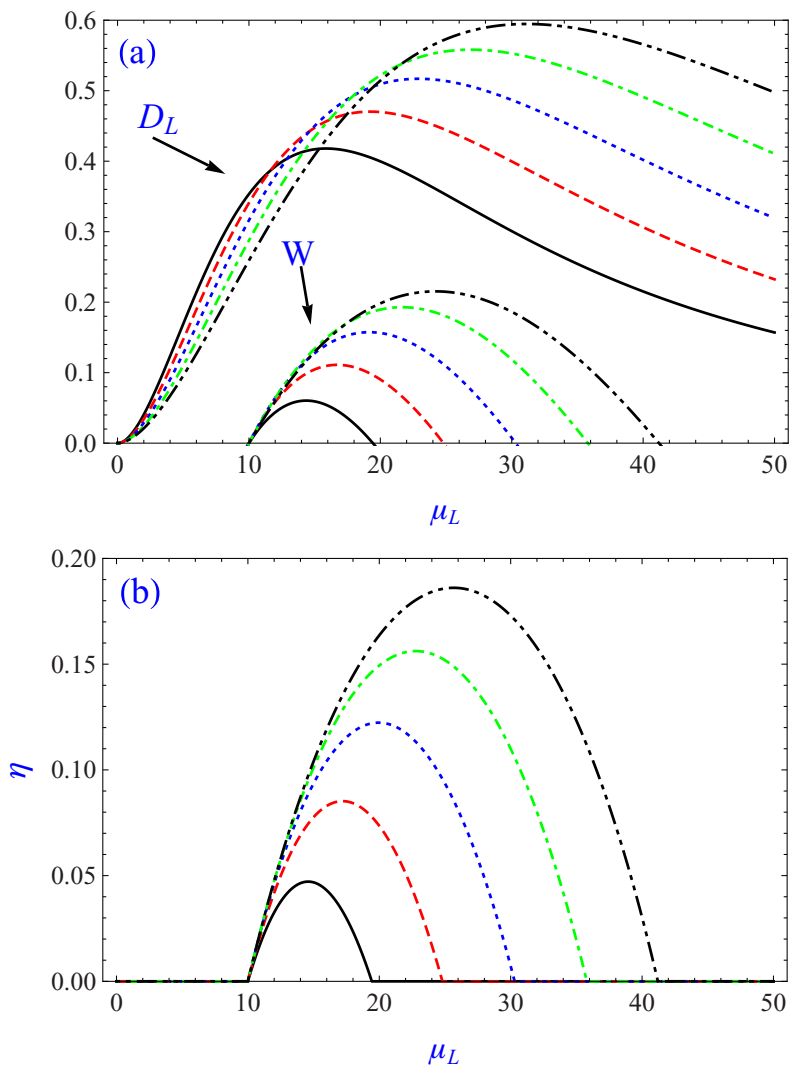

FIG. 7. (Color online) Dependence of positive work $W[(\mathrm{a})$, bottom bunch], $D_{L}$ [(a), top bunch], and efficiency $\eta$ (b) on $\mu_{L}$ for $k_{B} T_{H}=4, k_{B} T_{L}=1, \mu_{H}=10, \Omega=7$ (black, solid line), $\Omega=8$ (red, dashed line), $\Omega=9$ (blue, dotted line), $\Omega=10$ (green, dotdashed line), and $\Omega=11$ (black, dot-dot-dashed line). Note that $E_{L}$ is qualitatively same as $D_{L}$, so it is not plotted here. Also note that $D_{H}$ and $E_{H}$ increase as $\Omega$ increases.

A natural question which could arise is the possibility of work extraction when $\Omega_{H}<\Omega_{L}$ in the case of an interacting working substance with nonzero $\mu_{H}=\mu_{L}=\mu$, and when $\mu_{H}<\mu_{L}$ in the case of nonzero external field $\Omega_{H}=\Omega_{L}=\Omega$. The former case is not possible, since nonzero $\mu$ introduces just a shift to the energy levels, and the structure of the energy gaps remains the same. For the latter case, we can find narrowing gaps which are $E_{2}-E_{1}$ and $E_{4}-E_{3}$, as can be seen in Fig. 1(b), as $\mu_{H}$ is increased to $\mu_{L}$ in stage 2 of the cycle. For the small temperature limit or for large system parameters, the levels $E_{1}$ and $E_{2}$ are well separated from the others, and the contribution of their gap to work extraction is dominant. Accordingly, it would be possible to harvest positive work from the engine for $\mu_{H}<\mu_{L}$. Moreover, this effect arises in the deep quantum regime of the strongly interacting working medium so that the quantum correlations are expected to play a decisive role. Indeed, the maximum of the QD, positive work, and efficiency appear almost at the same $\mu_{L}$ in Fig. 7, beyond which they all decrease. The presence of nonzero $\Omega$ increases the QD, work, and efficiency. The machine produces work efficiently for the case $\mu_{L}>\mu_{H}\left(\mu_{H}=10\right.$ in Fig. 7) where this situation cannot be achieved in the absence of a magnetic field. Similar interplay of QD, work, and efficiency is found for the EoF, $E_{L}$, as well.

\section{COMPARISON OF RESULTS WITH PREVIOUS STUDIES}

A one-dimensional isotropic Heisenberg model of two spin-1/2 systems under a constant magnetic field has been considered as a working medium in a quantum Otto cycle in Ref. [35]. Only the magnetic field has been altered during the adiabatic stages of the cycle. It is found that if the magnetic field increases during the adiabatic expansion stage, then work cannot be extracted unless the working substance is interacting. If the working substance is interacting, then the work can be harvested for both the cases of increasing or decreasing of the magnetic field during the adiabatic stages. A condition of efficiency of the interacting working substance to be greater than the noninteracting one in terms of the magnetic fields at the end of adiabatic stages and temperatures of the heat reservoirs is given.

In our case, we consider an interacting working substance as well. The interaction is not of Heisenberg model but a singleaxis twisting spin squeezed one. Similar to Ref. [35], there is an external magnetic field as well. On the other hand, we assume not only magnetic field alteration during the adiabatic stages but examine the case of changing interaction strength during the adiabatic stages as well.

In contrast to Ref. [35], work cannot be extracted from our system if the magnetic field increases during the adiabatic expansion stage even for the interacting working substance. Work can be harvested only for decreasing magnetic field during the adiabatic expansion stage for both the interacting and noninteracting cases. If the interaction strength increases during the adiabatic expansion stage, a working substance cannot yield positive work if there is no external magnetic field. In the presence of an external magnetic field, however, work can be extracted for both the increasing or decreasing interactions during the adiabatic expansion stage.

Qualitative behavior of efficiency with the interaction strength shows significant differences between our model and the model of Ref. [35]. Efficiency increases slowly with interaction, then drops sharply after it reaches its maximum value in Ref. [35]. In our case, efficiency sharply reaches its maximum, then decreases slowly with the interaction. In addition, we examined the effect of altering interaction strength during the adiabatic stages, and it turned out to be more efficient than altering the magnetic field.

We examined the interplay of quantum correlations in terms of EoF and QD. Reference [35] does not examine quantum correlations. This brings new perspectives and insight and could be employed to the model of Ref. [35]. Some other studies already follow such an approach for the Heisenberg model; below we shall compare our results with them as well.

Influence of entanglement on the thermodynamic characteristics of a quantum Otto engine has been studied in Refs. [10,20,21]. A two-spin system with Heisenberg and Dzyaloshinski-Moriya interactions is considered in Ref. [20]. A two-spin isotropic $X X X$ model under constant magnetic field is considered in Ref. [21]. A cavity-mediated effective dipoledipole interaction of two spins in an optical cavity is considered 
in Ref. [10]. These studies examine the thermodynamic efficiency and positive work conditions of quantum Otto engines analytically and numerically. They also calculate the concurrence to characterize the quantum entanglement. It is reported that work cannot be extracted if the entanglements are the same at the end of thermalization stages [20,21]. Work can be extracted even if there is no entanglement at the end of thermalization with the hot reservoir. It is necessary to have entanglement at the end of thermalization with the cold reservoir in order to harvest work. There is a maximum work at a critical amount of entanglement at the end of the cold reservoir stage. Efficiency decreases with the entanglement at the end of the thermalization stage with the cold reservoir. Efficiency increases as the difference between the entanglements at two thermalization stages decreases.

In our case, we have a different physical model for the working substance, and we consider changing either the interaction or the magnetic field during the adiabatic stages, and in addition to quantum entanglement we examine quantum correlations beyond entanglement by QD. In contrast to Ref. [20], we find positive work even without any entanglement while QD is present. We find that entanglement at the end of thermalization stage with the hot bath is completely destructive for the work extraction if interaction is decreased in the adiabatic expansion stage or not changed. These studies [20] indicate that for small interactions or magnetic fields, the entanglement at the end of cold bath stage should be greater than the one at the end of the hot bath stage in order to extract work. For relatively large interactions or magnetic field strengths, positive work can be found irrespective of which entanglement is larger. In our case, entanglement at the end of the hot bath stage forbids work extraction, but QD behaves similar to these observations. For small interaction strengths, $\mathrm{QD}$ at the end of the cold bath stage is larger than the one at the end of the hot bath stage. For relatively larger interactions, positive work is found irrespective of which QD is larger. We find a maximum work at a critical interaction strength which is close to the location of maximum QD. Maximum work is harvested close to the maximum difference between the QDs for cold and hot bath thermalization stages.

It is found that there is a maximum efficiency at critical amounts of entanglement at the end of thermalization stages [21]. The relation between efficiency and entanglement is also nonmonotonic in our case. Efficiency vanishes if there is entanglement at the end of the thermalization stage with the hot reservoir. We find parameter regimes where QD exhibits qualitatively similar behavior with the work and efficiency. Maximum work and efficiency and QD can be simultaneously found at the same critical system parameters in our case. Reference [20] reports that high efficiency is possible for their model if entanglement is low but nonzero at the end of the thermalization stage with the cold reservoir. Entanglement at the end of the hot reservoir stage is not required, but if it is close to the one at the end of cold bath stage, then the efficiency is high. Our results suggests that strong quantum correlations beyond entanglement can yield high efficiency.

We also note that Ref. [9] also considers a quantum Otto engine and examines the effects of reservoir squeezing on the efficiency of work extraction. In our case squeezing is within the working substance, not in the reservoirs.

\section{CONCLUSIONS}

In summary, we proposed a four-level, correlated quantum Otto engine based on a one-axis twisting spin squeezing model with an external magnetic field. We discussed the work extraction from the engine and its efficiency as well as the role of quantum correlations, characterized by the quantum discord (QD) and the entanglement of formation (EoF) in the thermodynamical processes.

In our interacting spin Otto engine, a quantum working substance (quantum fuel) is generated by a particular nonlinear spin-spin interaction known as an axis-twisting spin squeezing interaction. This interaction yields quantum states of the bipartite spin system having pairwise entanglement as well as quantum correlations beyond entanglement, which can be characterized by a measure called QD.

The quantum fuel is cooled to low temperatures and compressed by a quantum adiabatic process in the preparation stages of the Otto cycle. These are described as the third and the fourth stages in our case. Compression means larger energy gaps for the quantum fuel. At low temperatures with strong interactions the quantum fuel is prepared with a quantum discord $D_{L}$. The cycle continues with the stages one and two where the fuel is burnt and expanded by another quantum adiabatic process to yield positive work. The expansion stage decreases the energy gaps. The system loses its quantumness and increases its classicality. When the quantum fuel is burnt, its QD is reduced to $D_{H}$. This could be interpreted as using the quantum information gradient as a resource, similar to the thermal gradient, to be further harvested by the engine to enhance positive work. We find that whether QD or entanglement can be interpreted as a resource or if they are just by-products depend on the parameter regimes. Further fundamental work is needed to rigorously justify if quantum correlations could indeed be used as a resource or not.

We can generally state the following conclusions for our model. Work cannot be harvested without a spin squeezing interaction if the magnetic field is decreased during the adiabatic expansion stage. Work cannot be harvested even with a spin squeezing interaction if the magnetic field increased during the adiabatic expansion stage. If the interaction is increased or decreased during the adiabatic expansion stage, then work can be harvested if there is an external magnetic field. The cycle can yield maximum work at maximum efficiency at critical interaction and magnetic field strengths. Variation of the magnetic field during the adiabatic stages instead of interaction yields larger efficiency and positive work, and the efficiency can be very close to the Carnot efficiency.

Entanglement of the working substance in the hot bath, $E_{H}$, forbids the work extraction if the external field or the interaction is decreased in the adiabatic expansion stage. In positive work regimes of these cases, $E_{L}>E_{H}=0$ and $E_{L}$ emerges after the maximum work, while QD is always present. Similarly $D_{L}>D_{H}$ for most cases except for the case of a changing magnetic field during the adiabatic stages, where small amount work can be extracted at large interaction strengths even when $D_{H}>D_{L}$. The relations between quantum correlations, work, and efficiency are nonmonotonic. Maximum work can be extracted at a critical magnetic field and interaction strengths at which $D_{L}$ is close to its maximum. This 
behavior is more pronounced if the interaction is increased during the adiabatic expansion stage. In this case positive work can be harvested at significantly large interaction strength, and quantum correlations induced by strong spin squeezing could play a more significant role. Work, efficiency, and $D_{L}$ have their maximums almost at the same critical system parameters. Moreover, entanglement $E_{L}$ behaves similar to $D_{L}$, and $E_{H}$ can be nonzero in a positive work regime in this case.

\section{ACKNOWLEDGMENTS}

The authors warmly thank N. Allen for fruitful discussions. F.A. acknowledges the support and hospitality of the Office of Vice President for Research and Development (VPRD) and Department of Physics of Koç University. Ö.E.M. acknowledges the support of a Lockheed Martin Corporation Grant. A.Ü.C.H. acknowledges COST Action MP1209.
[1] K. Maruyama, F. Nori, and V. Vedral, Rev. Mod. Phys. 81, 1 (2009).

[2] M. O. Scully, Phys. Rev. Lett. 104, 207701 (2010).

[3] F. Tonner and G. Mahler, Phys. Rev. E 72, 066118 (2005).

[4] H. P. Goswami and U. Harbola, Phys. Rev. A 88, 013842 (2013).

[5] A. E. Allahverdyan, R. S. Gracià, and T. M. Nieuwenhuizen, Phys. Rev. E 71, 046106 (2005).

[6] J. Wang, Z. Wu, and J. He, Phys. Rev. E 85, 041148 (2012).

[7] T. D. Kieu, Phys. Rev. Lett. 93, 140403 (2004).

[8] H. T. Quan, Y.-x. Liu, C. P. Sun, and F. Nori, Phys. Rev. E 76, 031105 (2007).

[9] X. L. Huang, T. Wang, and X. X. Yi, Phys. Rev. E 86, 051105 (2012).

[10] H. Wang, S. Liu, and J. He, Phys. Rev. E 79, 041113 (2009).

[11] M. Kitagawa and M. Ueda, Phys. Rev. A 47, 5138 (1993).

[12] X. Wang and B. C. Sanders, Phys. Rev. A 68, 012101 (2003).

[13] J. Ma, X. Wang, C. Sun, and F. Nori, Phys. Rep. 509, 89 (2011).

[14] C. Gross, T. Zibold, E. Nicklas, J. Esteve, and M. K. Oberthaler, Nature (London) 464, 1165 (2010).

[15] M. F. Riedel, P. Böhi, Y. Li, T. W. Hänsch, A. Sinatra, and P. Treutlein, Nature (London) 464, 1170 (2010).

[16] W. K. Wootters, Phys. Rev. Lett. 80, 2245 (1998).

[17] H. Ollivier and W. H. Zurek, Phys. Rev. Lett. 88, 017901 (2001).

[18] L. Henderson and V. Vedral, J. Phys. A: Math. Gen. 34, 6899 (2001).

[19] K. Modi, A. Brodutch, H. Cable, T. Paterek, and V. Vedral, Rev. Mod. Phys. 84, 1655 (2012).
[20] G.-F. Zhang, Eur. Phys. J. D 49, 123 (2008).

[21] T. Zhang, W.-T. Liu, P.-X. Chen, and C.-Z. Li, Phys. Rev. A 75, 062102 (2007)

[22] K. V. Hovhannisyan, M. Perarnau-Llobet, M. Huber, and A. Acín, Phys. Rev. Lett. 111, 240401 (2013).

[23] N. Brunner, M. Huber, N. Linden, S. Popescu, R. Silva, and P. Skrzypczyk, Phys. Rev. E 89, 032115 (2014).

[24] R. Alicki and M. Fannes, Phys. Rev. E 87, 042123 (2013).

[25] J. J. Park, K.-H. Kim, T. Sagawa, and S. W. Kim, Phys. Rev. Lett. 111, 230402 (2013).

[26] K. Funo, Y. Watanabe, and M. Ueda, Phys. Rev. A 88, 052319 (2013).

[27] L. A. Correa, J. P. Palao, G. Adesso, and D. Alonso, Phys. Rev. E 87, 042131 (2013).

[28] M. O. Scully and M. S. Zubairy, Quantum Optics (Cambridge University Press, Cambridge, 1997).

[29] R. Jozsa, J. Mod. Opt. 41, 2315 (2007).

[30] M. S. Sarandy, L.-A. Wu, and D. A. Lidar, Quantum Inf. Process. 3, 331 (2004).

[31] Y. Huang, New J. Phys. 16, 033027 (2014).

[32] F. F. Fanchini, T. Werlang, C. A. Brasil, L. G. E. Arruda, and A. O. Caldeira, Phys. Rev. A 81, 052107 (2010).

[33] Y. Huang, Phys. Rev. A 88, 014302 (2013).

[34] A. Ferraro, L. Aolita, D. Cavalcanti, F. M. Cucchietti, and A. Acín, Phys. Rev. A 81, 052318 (2010).

[35] G. Thomas and R. S. Johal, Phys. Rev. E 83, 031135 (2011). 